\title{
ArXives of Earth science
}

\author{
Preprint servers afford a platform for sharing research before peer review. We are pleased that two dedicated \\ preprint servers have opened for the Earth sciences and welcome submissions that have been posted there first.
}

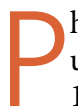
hysicists have been posting their unpublished papers on ArXiv.org since 1991. The subject areas covered have expanded over the years to include fields as diverse as economics, electrical engineering and mathematics. Submission rates have risen steeply, to over 120,000 papers per year in 2017 (https://arxiv.org/help/ stats/2017_by_area/index): scientists clearly find the service useful. Yet there has been no comparable resource for geoscientists. With the launch of the community-led EarthArXiv (https://eartharxiv.org) and the American Geophysical Union's Earth and Space Science Open Archive (ESSOAr; https://essoar.org/), that might be about to change. At Nature Geoscience, we do not count preprints in our assessment of the advance a submitted manuscript constitutes to the body of literature, and we welcome submissions that have previously been posted.

Formal peer review takes time. Manuscripts are carefully vetted by reviewers and they typically undergo several rounds of review and revision before they are ready to be published. Peer review is an important component of the publication process that helps ensure the integrity of the scientific record - but fast it is not. In an academic world structured around short grant cycles and temporary research positions whose procurement depends on a scholarly track record, there is room for a parallel route for disseminating the latest science findings that is more agile, but in turn less rigorously quality controlled.

Preprint servers fit this bill. Manuscripts are freely uploaded, become immediately shareable and are assigned a digital object identifier (doi) that many funding agencies and prospective employers will consider in grant proposals and job applications. The papers are also open to comment from the community, and preprint servers are billed as another avenue for debate, testing and validation of papers. The idea of community-driven, open peer review has potential. Relevant experts can find, read and thoroughly review pertinent papers on preprint servers, with all comments and responses posted openly. In practice, however, the sheer volume of research papers produced each year makes it unlikely that each preprint will glean an appropriate amount of community attention and scrutiny. For example, in 2017 Nature Geoscience received 2,092 new manuscript submissions.

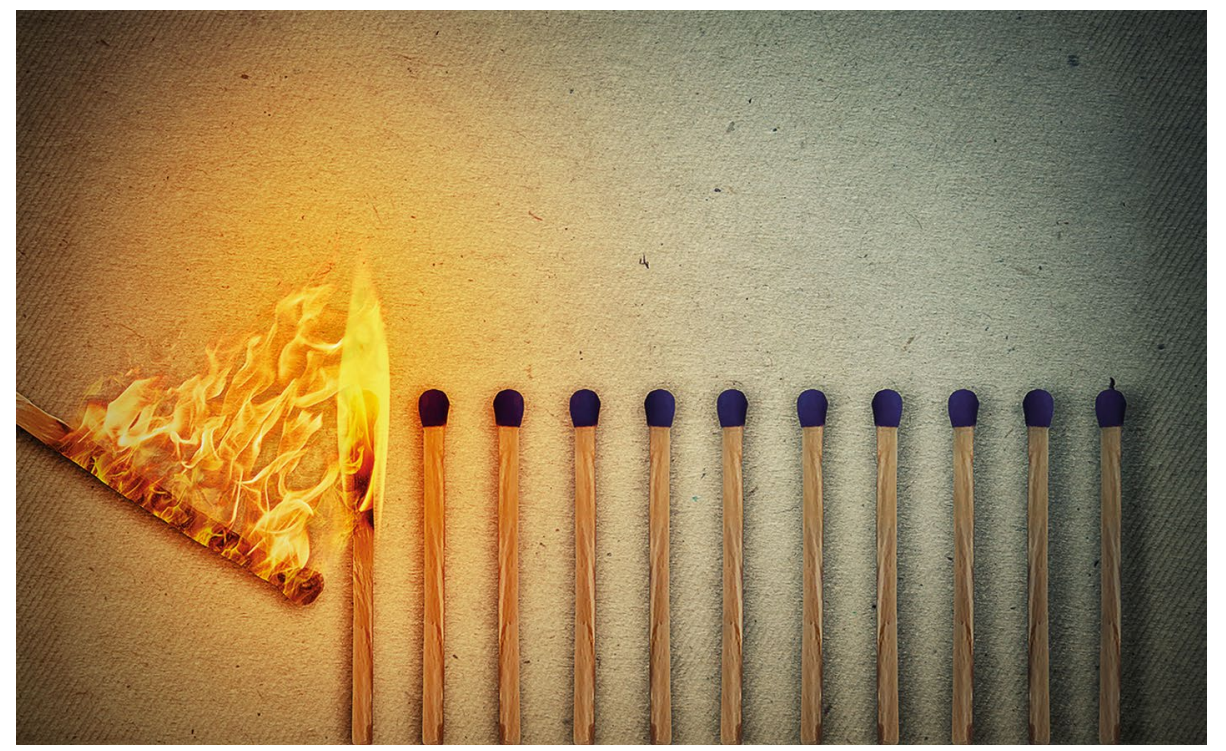

Preprint servers can help expedite the scientific spark. Credit: Bordeianu Andrei / Alamy Stock Photo

We sent out to review 361 articles and each one was assigned to an average of 2.9 referees. This means that more than 1,000 academics reviewed for us last year. We asked closer to 2,000 people to review them (an average of about five reviewers per paper), and the most commonly cited reason for declining review was simply a lack of time, with three-quarters of negative respondents saying they were too busy or travelling.

The submissions to Nature Geoscience represent just a small sample of the research produced by the Earth and planetary science communities every year - EarthArxiv and ESSOAr will eventually host many times this volume. It is not clear that all of these preprints will be read: without an editor that solicits reviews or heuristics such as journal reputation to decide which papers to look at, it will largely be up to the authors to find an audience for their paper, which runs the risk of articles being read mostly by friends and colleagues.

Indeed, a quick browse of the European Geosciences Union's discussions journals (https://www.egu.eu/publications/ open-access-journals/) - which place submissions in the public domain, in parallel to solicited peer review, to allow the community to comment - reveals that comments on papers are largely limited to those from the solicited reviewers. It seems likely that under those circumstances, it is not the quality of the science but the authors' connections and the reputation of their institutions that will be the most important factor in attracting readers - and ultimately rewards for the authors.

Of course, the peer-review system is by no means free of such bias. At Nature Geoscience, we offer double-blind peer review to help remove unconscious bias from reviewer decisions, and as editors, we champion excellent submissions from anyone, regardless of their connections. We hope that this will help place research selected for its scientific impact in front of a broad audience.

Other disciplines have found the fast dissemination of research by publication of preprint valuable. We support the use of these repositories for this purpose.

In addition, in line with our green open-access policy (https://www.nature.com/authors/ policies/license), we encourage authors to make the accepted version of their paper available on preprint servers six months after publication, so that the record is completed.

It will be interesting to see whether Earth scientists will pick up on the benefits of using preprint servers, now that dedicated options are available.

Published online: 28 February 2018 https://doi.org/10.1038/s41561-018-0083-y 